\title{
Development of an Efficient Synthesis of a Key Intermediate for Baloxavir Marboxil from a Greener Start: Ethylene Glycol
}

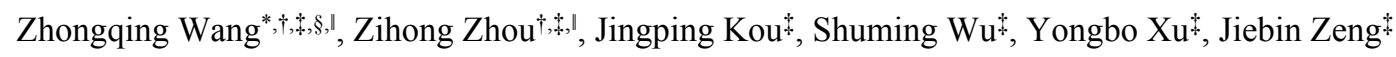

†State Key Laboratory of Anti-Infective Drug Development (NO. 2015DQ780357), Sunshine Lake Pharma Co., Ltd, Dongguan 523871, P. R. China

Department of Process Research and Development, HEC Pharm Group, Dongguan 523871, P. R. China

${ }^{\S}$ School of Pharmacy, Xiangnan University, Chenzhou 423000 Hunan, China

*E-mail:Wangzhongqing@hec.cn

\section{Supporting Information}

Table of Contents

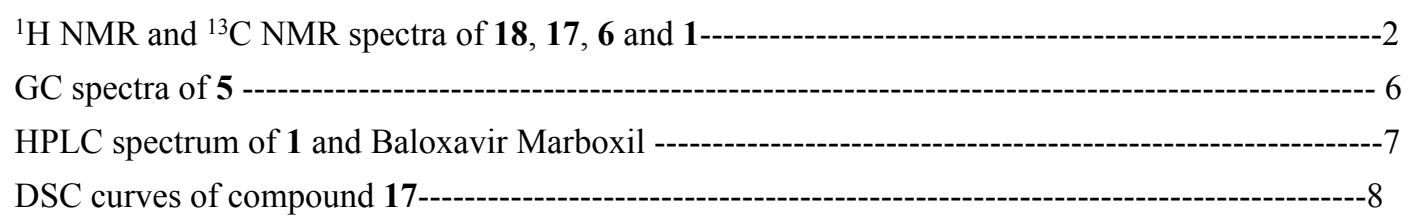


${ }^{1} \mathrm{H}$ NMR and ${ }^{13} \mathrm{C}$ NMR spectra of $18,17,6$ and 1

${ }^{1} \mathrm{H}$ NMR spectra of $\mathbf{1 8}$

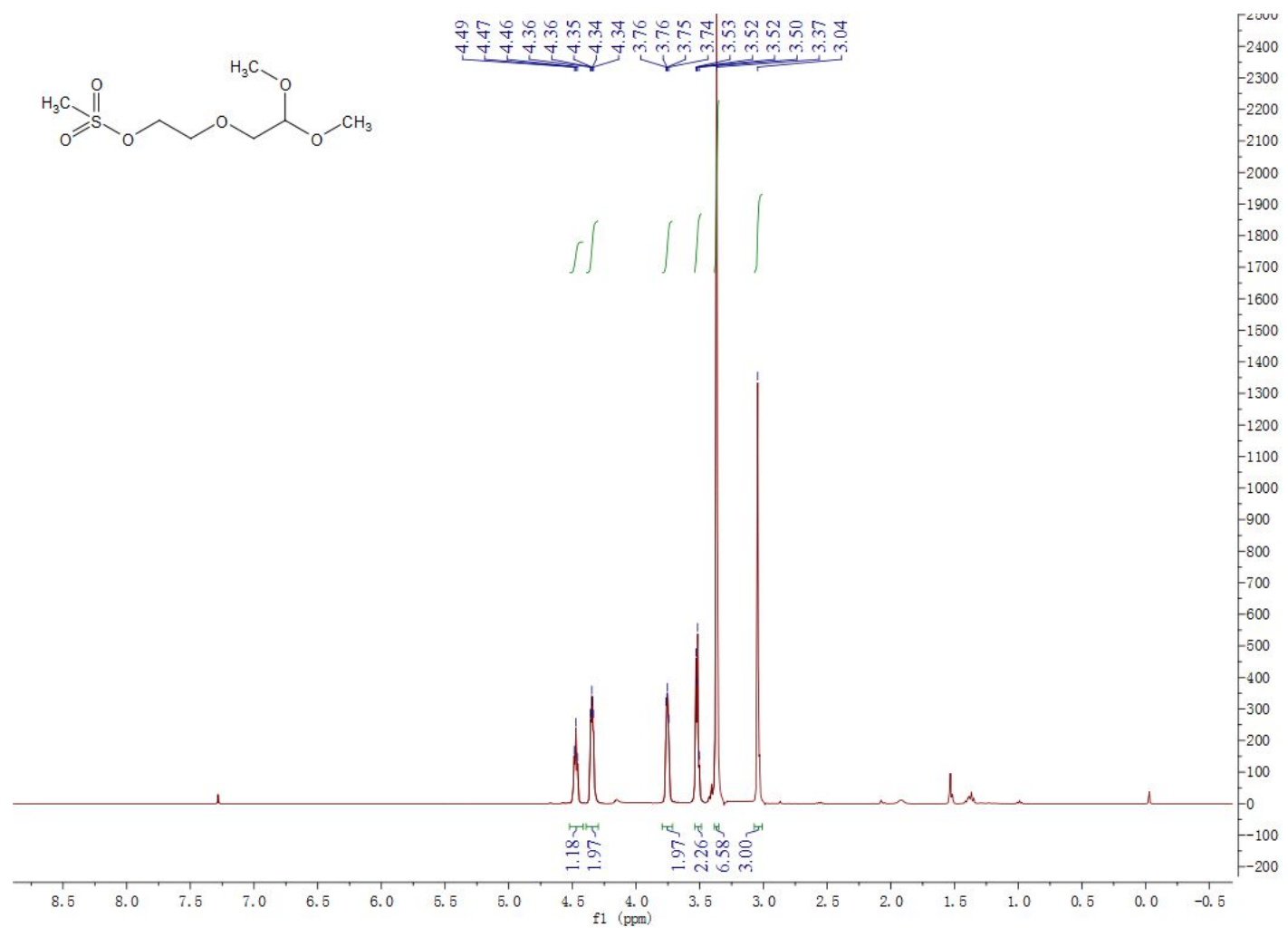

${ }^{13} \mathrm{C}$ NMR spectra of $\mathbf{1 8}$

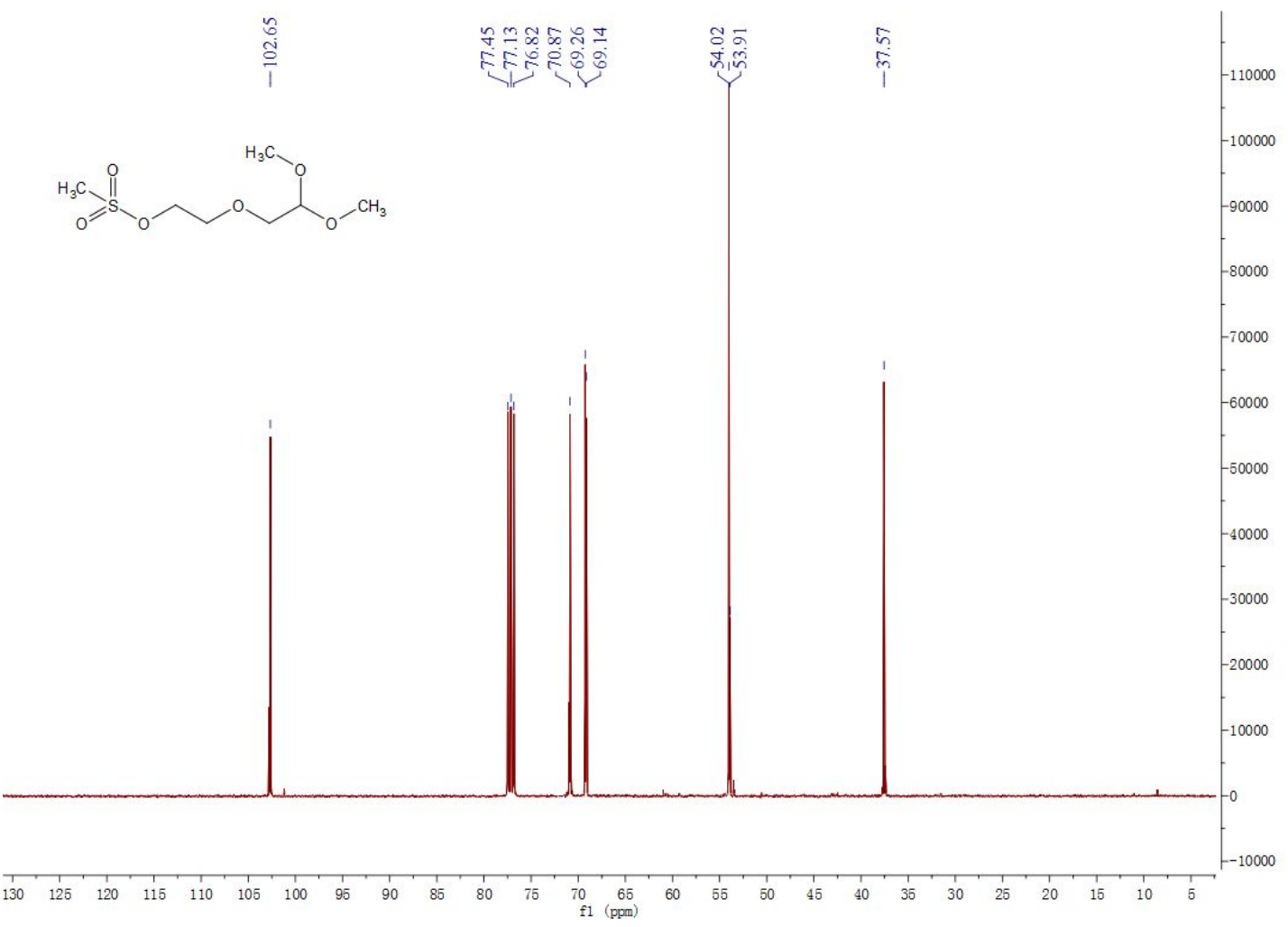


${ }^{1} \mathrm{H}$ NMR spectra of $\mathbf{1 7}$

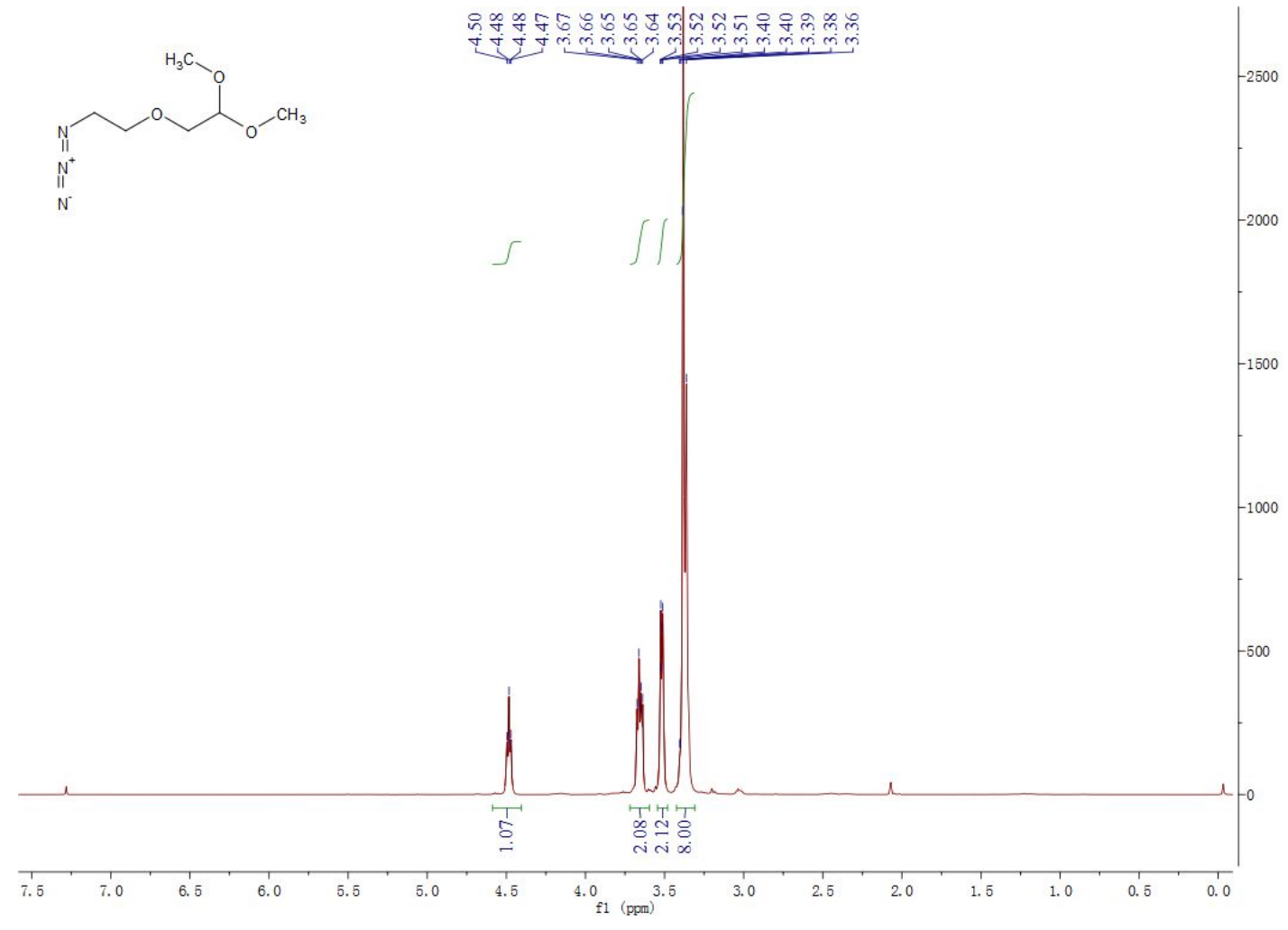

${ }^{13} \mathrm{C}$ NMR spectra of $\mathbf{1 7}$

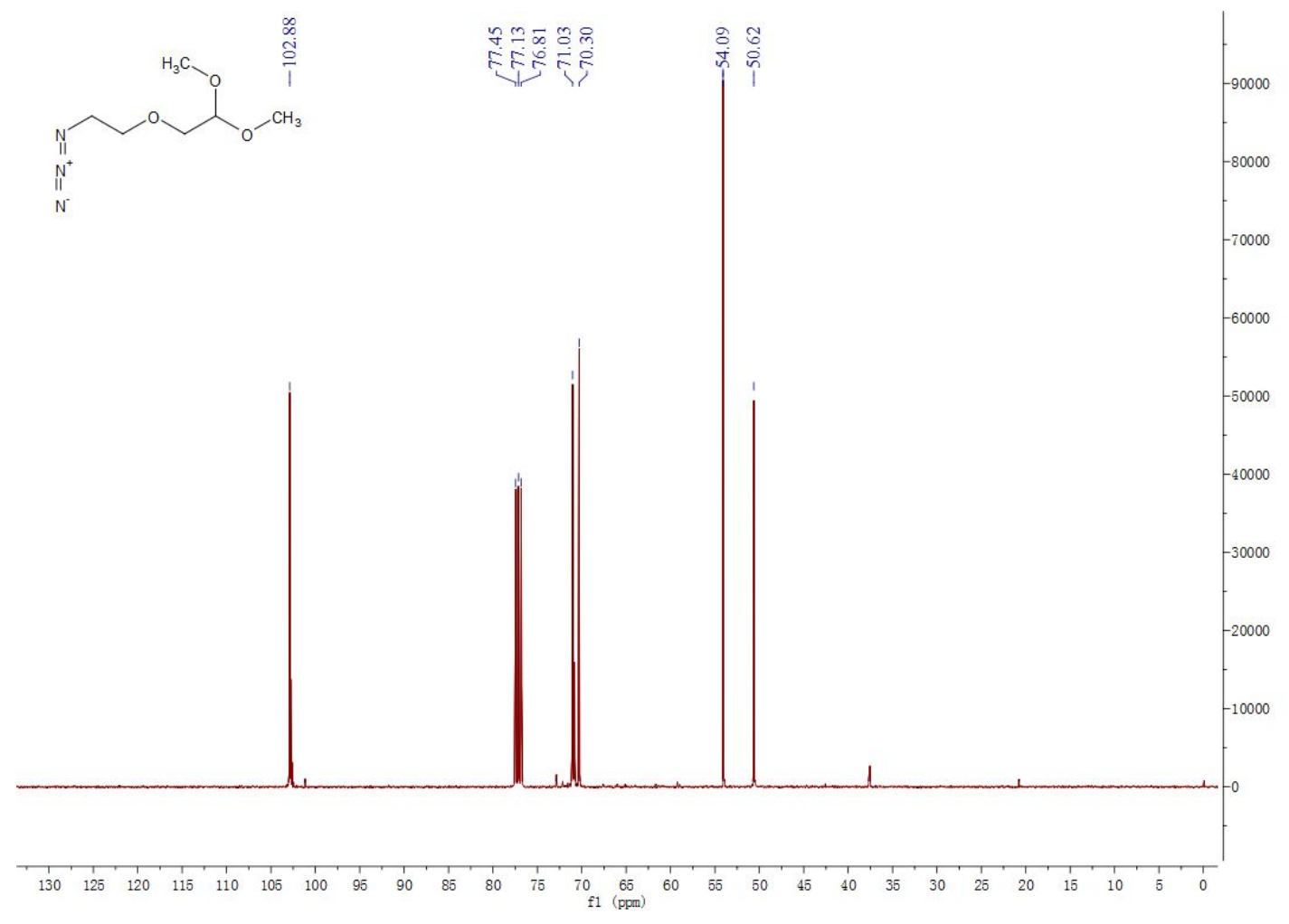


${ }^{1} \mathrm{H}$ NMR spectra of 6

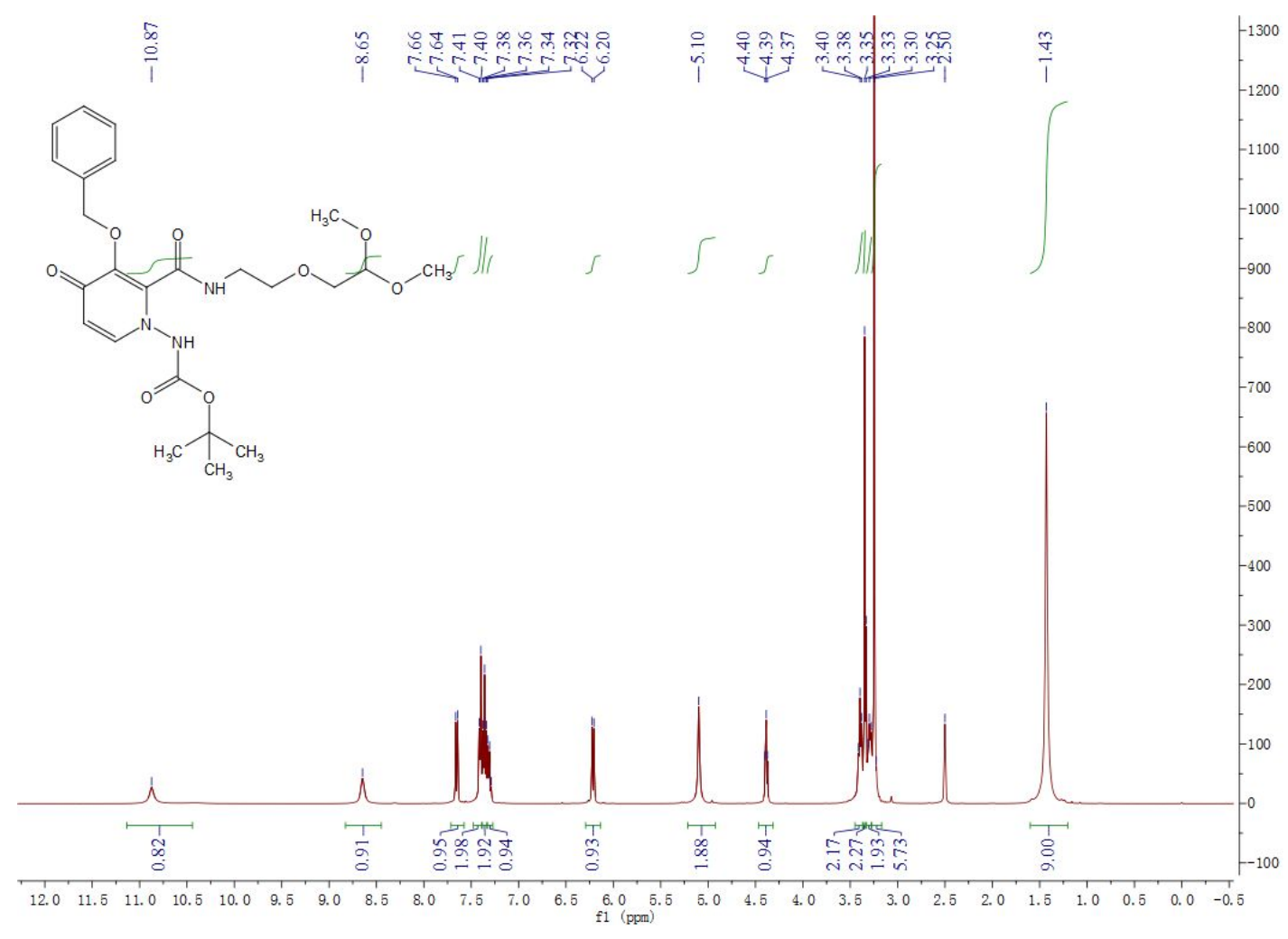

${ }^{13} \mathrm{C}$ NMR spectra of 6

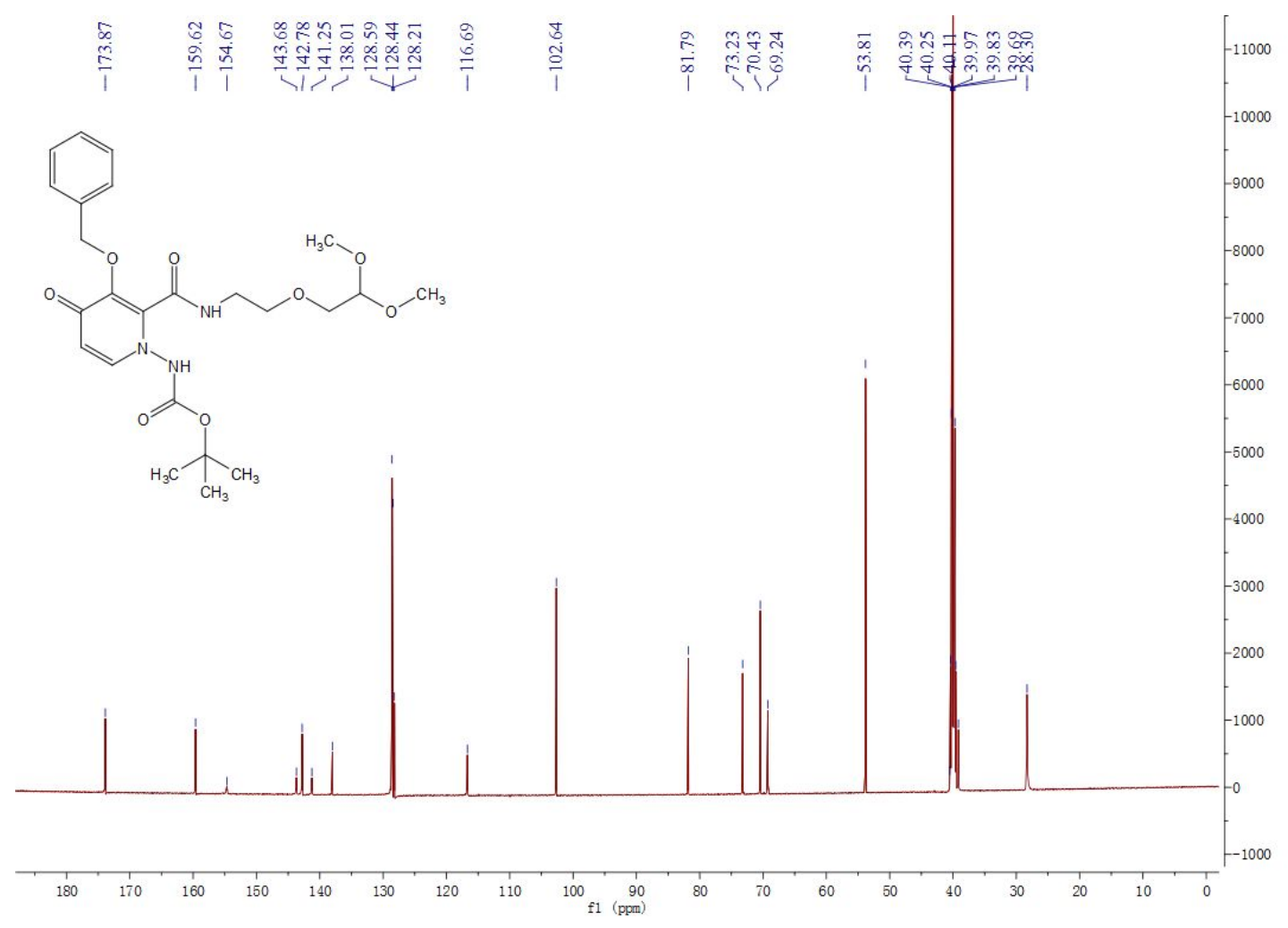

4 

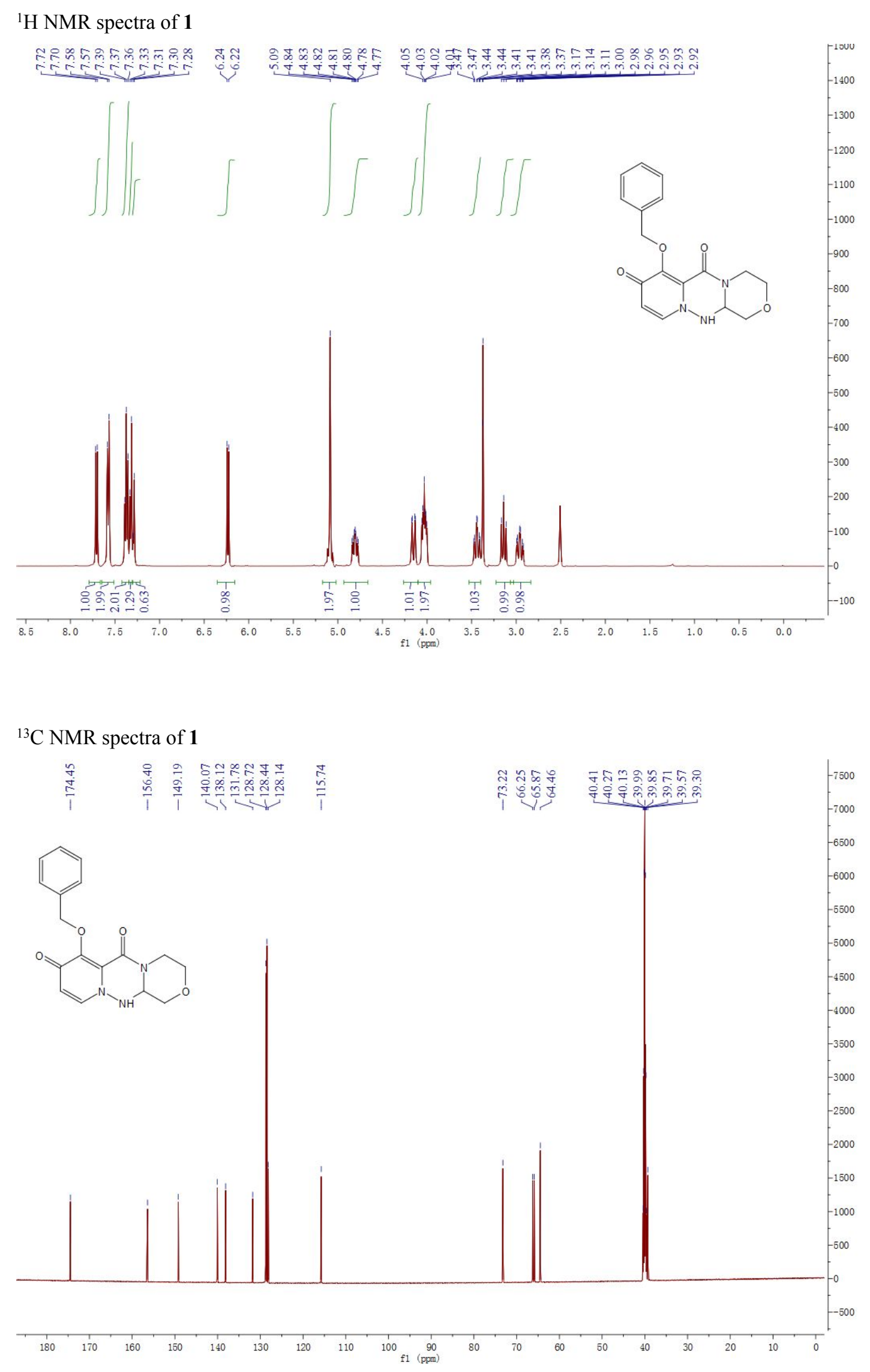

5 


\section{GC spectra of 5}

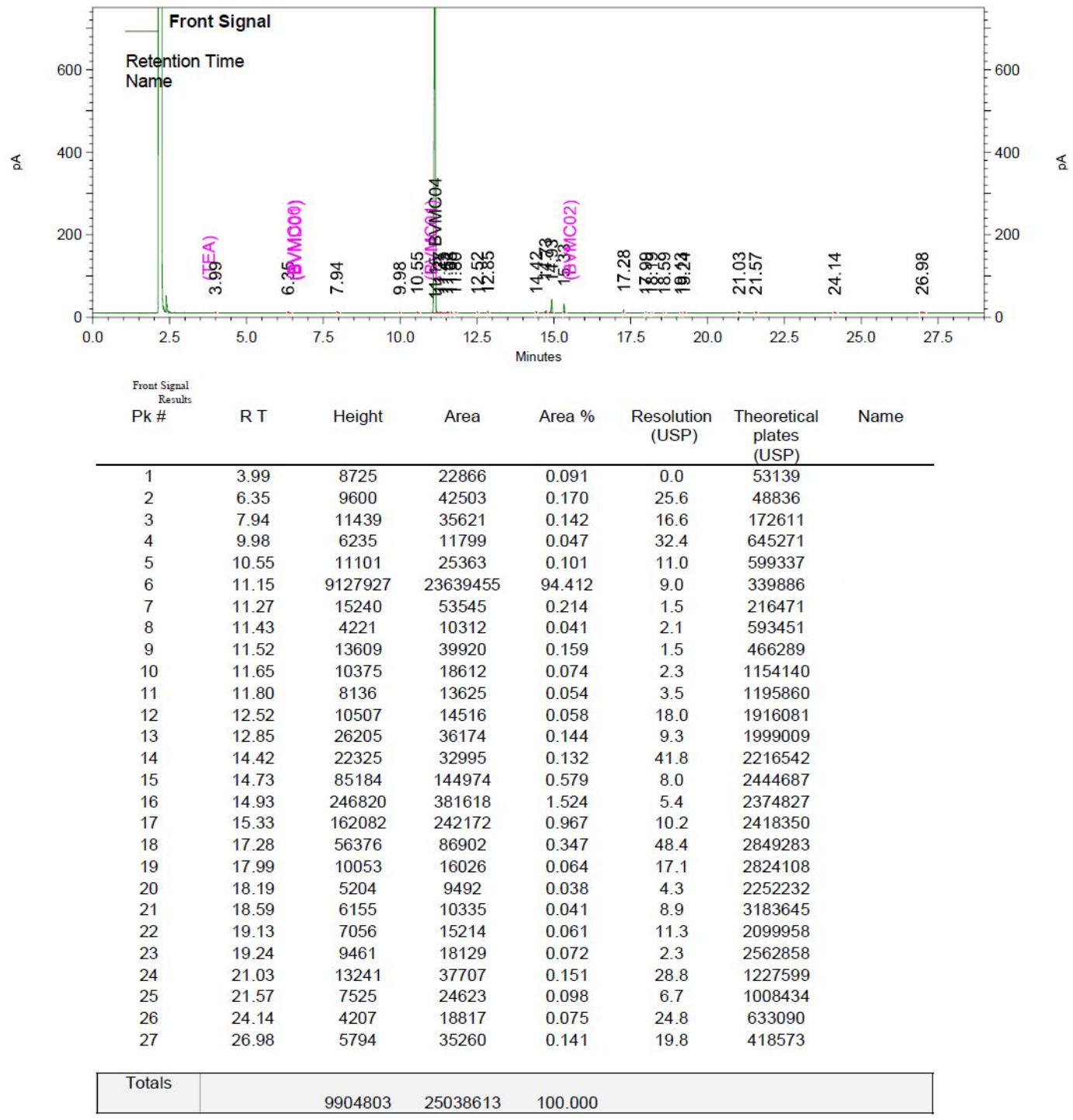

Chromatography System: Agilent OpenLAB CDS (EZChrom Edition) Version A.04.05 
HPLC spectrum of $\mathbf{1}$ and Baloxavir Marboxil

HPLC spectrum of 1

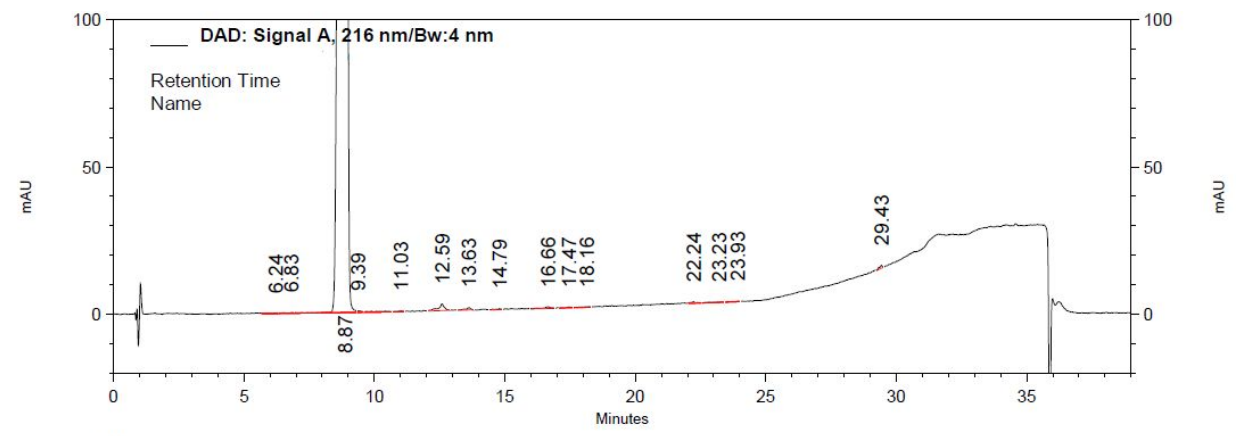

\begin{tabular}{|c|c|c|c|c|c|c|c|c|c|c|}
\hline $\begin{array}{r}\text { DAD: } \\
\text { Signal A, } \\
216 \\
\text { nm/Bw:4 } \\
\text { nm Results } \\
\text { Pk \# }\end{array}$ & R T & Height & Area & Area $\%$ & $\begin{array}{l}\text { Resolutio } \\
\mathrm{n} \text { (USP) }\end{array}$ & $\begin{array}{l}\text { Asym } \\
\text { metry }\end{array}$ & $\begin{array}{l}\text { Theoretical } \\
\text { plates (USP) }\end{array}$ & $\begin{array}{l}\text { Peak } \\
\text { purity }\end{array}$ & RRT & Name \\
\hline 1 & 6.24 & 444 & 10182 & 0.03 & 0.0 & 0.0 & 1576 & 1.000 & 0.00 & \\
\hline 2 & 6.83 & 647 & 11100 & 0.03 & 0.9 & 0.0 & 1351 & 1.000 & 0.00 & \\
\hline 3 & 8.87 & 2596261 & 37397360 & 99.30 & 3.8 & 0.7 & 10755 & 0.997 & 0.00 & \\
\hline 4 & 9.39 & 1142 & 11733 & 0.03 & 1.6 & 0.0 & 13983 & 1.000 & 0.00 & \\
\hline 5 & 11.03 & 712 & 11876 & 0.03 & 4.1 & 0.0 & 8589 & 1.000 & 0.00 & \\
\hline 6 & 12.59 & 4759 & 80414 & 0.21 & 3.3 & 0.7 & 11270 & 1.000 & 0.00 & \\
\hline 7 & 13.63 & 1694 & 23029 & 0.06 & 2.8 & 0.8 & 40555 & 1.000 & 0.00 & \\
\hline 8 & 14.79 & 741 & 10409 & 0.03 & 3.0 & 0.0 & 13872 & 1.000 & 0.00 & \\
\hline 9 & 16.66 & 1274 & 28556 & 0.08 & 4.1 & 0.0 & 25603 & 1.000 & 0.00 & \\
\hline 10 & 17.47 & 714 & 10886 & 0.03 & 0.0 & 0.0 & 0 & 1.000 & 0.00 & \\
\hline 11 & 18.16 & 559 & 14724 & 0.04 & 0.0 & 0.0 & 21724 & 1.000 & 0.00 & \\
\hline 12 & 22.24 & 1079 & 11142 & 0.03 & 0.0 & 0.0 & 0 & 1.000 & 0.00 & \\
\hline 13 & 23.23 & 409 & 13085 & 0.03 & 0.0 & 0.0 & 37850 & 1.000 & 0.00 & \\
\hline 14 & 23.93 & 612 & 10150 & 0.03 & 1.3 & 0.0 & 24258 & 1.000 & 0.00 & \\
\hline 15 & 29.43 & 2041 & 17117 & 0.05 & 14.3 & 0.7 & 566704 & 1.000 & 0.00 & \\
\hline Totals & & 2613088 & 37661763 & 100.00 & & & & & & \\
\hline
\end{tabular}

Chromatography System: Agilent OpenLAB CDS (EZChrom Edition) Version A.04.05

HPLC spectrum of Baloxavir Marboxil
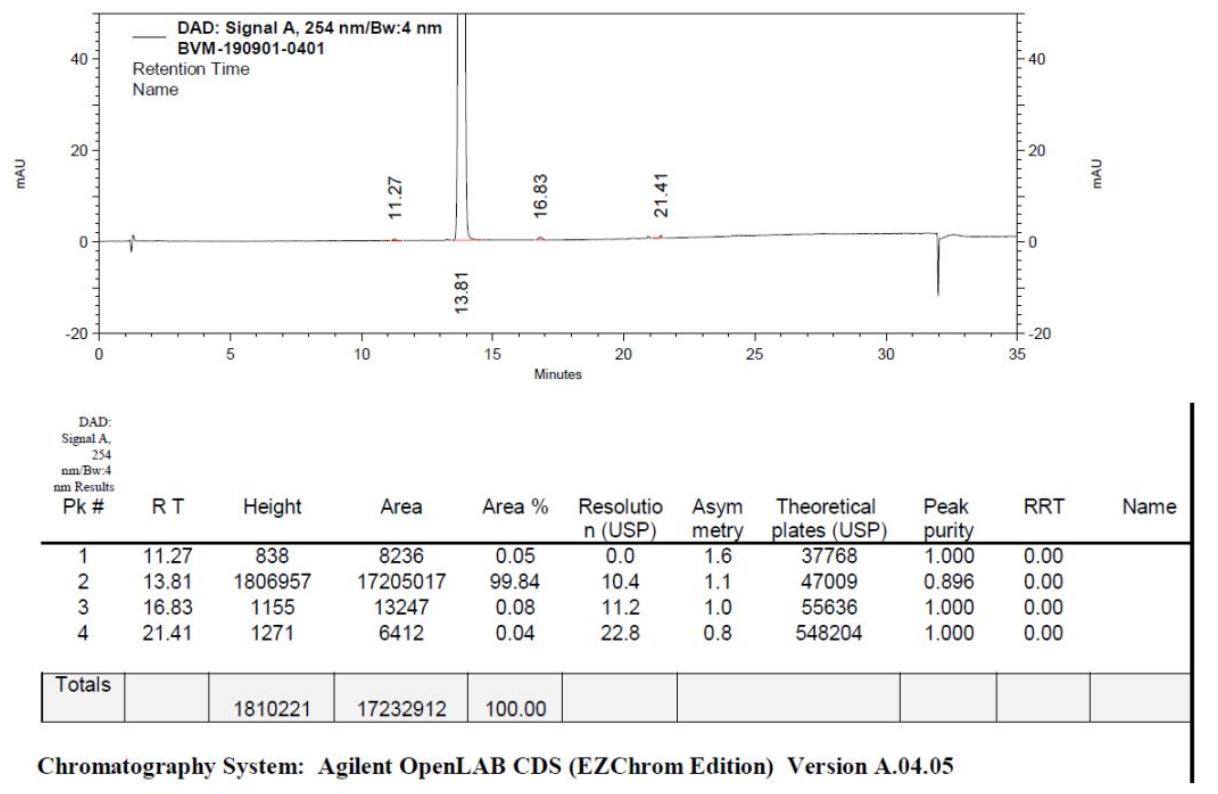
DSC curves of compound 17

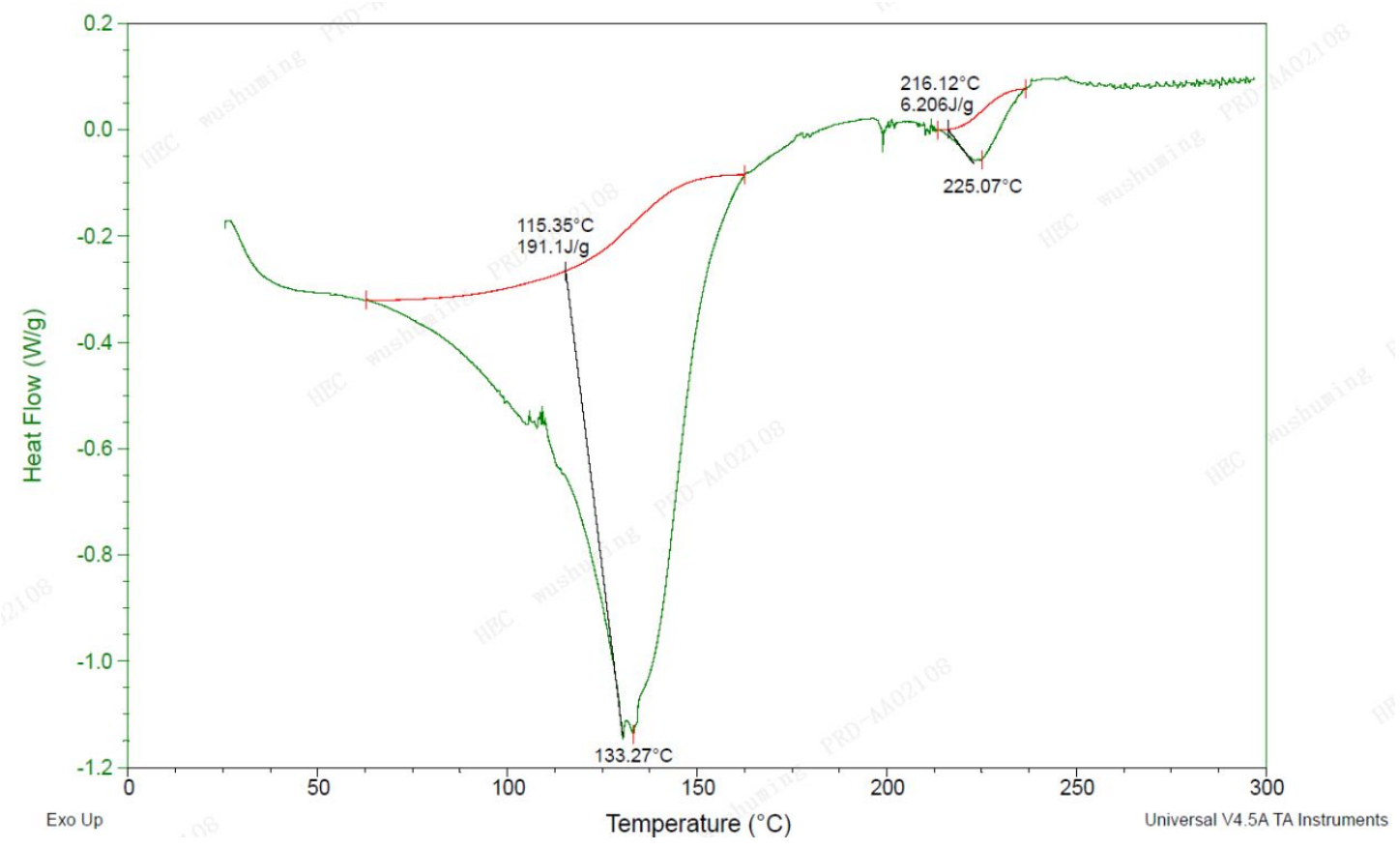

